\title{
Genetically modified Lactococcus lactis producing a green fluorescent protein-bovine lactoferrin fusion protein suppresses proinflammatory cytokine expression in lipopolysaccharide-stimulated RAW 264.7 cells
}

\author{
S. Shigemori, ${ }^{*} \dagger^{1}$ F. Namai, $¥$ Y. Yamamoto, ${ }^{*} \dagger$ S. Nigar, ${ }^{\star} \S$ T. Sato,\# T. Ogita,,$I$ and T. Shimosato* $\ddagger \| \eta^{2}$ \\ *Department of Bioscience and Food Production Science, Interdisciplinary Graduate School of Science and Technology, Shinshu University, \\ 8304 Minamiminowa, Kamiina, Nagano 3994598, Japan \\ †Research Fellow of the Japan Society for the Promotion of Science, Japan Society for the Promotion of Science, 5-3-1, Kojimachi, Chiyoda-ku, \\ Tokyo 1020083, Japan \\ ‡Department of Agricultural and Life Science, Graduate School of Science and Technology, Shinshu University, 8304 Minamiminowa, Kamiina, \\ Nagano 3994598, Japan \\ $\S$ Department of Nutrition and Food Technology, Jessore University of Science and Technology, Churamonkathi-Chowgacha Rd, Jessore 7408, \\ Bangladesh \\ \#Department of Pulmonology, Graduate School of Medicine, Yokohama City University, 3-9 Fukuura, Kanazawa-ku, Yokohama, \\ Kanagawa 2360004, Japan \\ IIDepartment of Interdisciplinary Genome Sciences and Cell Metabolism, Institute for Biomedical Sciences, Shinshu University, \\ 8304 Minamiminowa, Kamiina, Nagano 3994598, Japan \\ 『Research Center for Fungal and Microbial Dynamism, Shinshu University, 8304 Minamiminowa, Kamiina, Nagano 3994598, Japan
}

\section{ABSTRACT}

Lactoferrin (LF), an iron-binding glycoprotein distributed widely in the biological fluids of mammals, is believed to play an important role in host defenses against infection. Previous studies in animal models and humans demonstrated that combined administration of LF and probiotic lactic acid bacteria (LAB) can prevent sepsis. In this study, we genetically engineered a probiotic LAB strain, Lactococcus lactis, to produce recombinant bovine LF based on the green fluorescent protein (GFP)-fused expression system. Western blotting confirmed that the genetically modified $L$. lactis strain (designated NZ-GFP-bLF) produced a protein corresponding to a fusion of GFP and bLF in the presence of nisin, an inducer of target gene expression. The protein synthesized by NZ-GFP-bLF was fluorescent and thus we monitored the time-dependent change in the production level of the recombinant protein using fluorometric analysis. The utility of NZ-GFP-bLF in preventing sepsis was determined by investigating its anti-inflammatory property in lipopolysaccharide

\footnotetext{
Received March 15, 2017.

Accepted May 12, 2017.

${ }^{1}$ Current affiliations: Department of Intestinal Ecosystem Regulation, Faculty of Medicine, University of Tsukuba, 1-1-1, Tennodai, Tsukuba, Ibaraki 3058575, Japan. Metabologenomics Core, Transborder Medical Research Center, University of Tsukuba, 1-1-1, Tennodai, Tsukuba, Ibaraki 3058575, Japan.

${ }^{2}$ Corresponding author: shimot@shinshu-u.ac.jp
}

(LPS)-stimulated mouse macrophage RAW 264.7 cells. Pretreatment of RAW 264.7 cells with NZ-GFP-bLF significantly attenuated the LPS-induced mRNA expression and protein production of 3 proinflammatory cytokines (IL-1 $\alpha$, IL-6, and tumor necrosis factor- $\alpha$ ) compared with pretreatment with a vector control strain of L. lactis. Our results suggest that NZ-GFPbLF holds promise for the development of a new prophylaxis for sepsis.

Key words: lactoferrin, green fluorescent protein, Lactococcus lactis, lipopolysaccharide

\section{INTRODUCTION}

Lactoferrin $(\mathbf{L F})$ is an iron-binding glycoprotein that is widely distributed in the biological fluids of mammals (Siqueiros-Cendón et al., 2014). Lactoferrin is present in especially high concentration in milk, and its concentration in colostrum from human and cow is 7 and $2 \mathrm{~g} / \mathrm{L}$, respectively (Masson and Heremans, 1971; Tsuji et al., 1990). Lactoferrin is believed to play an important role in innate immune host defenses against infection. Indeed, previous studies have demonstrated that LF exerts bacteriostatic and bactericidal activities through various mechanisms, such as iron sequestration from the bacterial growth environment, attributed to its iron-binding ability, and direct interaction of LF with the bacterial surface to induce bacterial lysis (Farnaud and Evans, 2003). Furthermore, LF beneficially modulates excessive immune responses induced by LPS, a major pathogenic factor of gram-negative bacteria, in mammalian cells via direct interaction with LPS 
or with the host cells (Latorre et al., 2010). Lactoferrin therefore holds promise as a natural agent against infectious diseases, including sepsis. Indeed, in vivo studies with animal models have demonstrated that LF administration protects from lethal shock (Zagulski et al., 1989; Lee et al., 1998; Kruzel et al., 2000), hepatitis (Zagulski et al., 1989), and diarrhea (Talukder and Harada, 2007) induced by LPS or pathogenic bacteria. Interestingly, an earlier study using rat pups found that oral administration of LF exerts an antibacterial effect against invasive Escherichia coli infection in the intestine, and this effect was enhanced by co-administration of a probiotic lactic acid bacteria (LAB) strain, Lactobacillus rhamnosus GG (Sherman et al., 2004). Based on these findings, a clinical study was conducted to evaluate the effectiveness of bovine LF (bLF) plus/ minus probiotics regimens in low-birth-weight neonates and demonstrated the significant preventive effect on late-onset sepsis of these regimens compared with placebo treatment (Manzoni et al., 2009).

The co-administration of LF and probiotic LAB may be effective for preventing infections; thus, genetically modified (gm)LAB engineered to produce LF could be of medical use. Genetically modified LAB strains producing different biomedical proteins have been engineered over the past 2 decades as a mucosal delivery vehicle for proteins. In addition, numerous studies have suggested the usefulness of mucosal application of gmLAB strains for the prevention and therapy of various disorders, such as infections, allergies, and inflammatory diseases (Wells and Mercenier, 2008; Cano-Garrido et al., 2015; Shigemori and Shimosato, 2017). For example, our previous study demonstrated that a genetically modified strain of Lactococcus lactis secreting bioactive heme oxygenase-1, an enzyme with anti-inflammatory and cell protective properties, can deliver the recombinant protein to the colon following oral administration and helps alleviate dextran sulfate sodium-induced acute colitis in mice (Shigemori et al., 2015).

The aim of the present study was to develop a new gmLAB strain producing bLF as a candidate strain for the prevention of sepsis. We therefore engineered a Lactococcus lactis strain to express a recombinant bLF gene based on the green fluorescent protein (GFP) fusion system. This is a modification of a gene expression system in L. lactis established previously by us that allows for rapid, simple, and accurate measurement of target protein expression (Shigemori et al., 2012, 2013). Additionally, we compared the anti-inflammatory properties of the recombinant bLF-producing and non-bLFproducing strains of genetically modified L. lactis in an LPS-stimulated mouse macrophage cell line, RAW 264.7 .

\section{MATERIALS AND METHODS}

\section{Bacterial Strains and Growth Conditions}

Lactococcus lactis NZ9000 (NZ9000; MoBiTec, Gottingen, Germany) was used as a host strain for gene expression; NZ-VC (Shigemori et al., 2012) is an NZ9000 strain harboring the lactococcal gene expression vector pNZ8148\#2:CYT and was used as a vector control strain. The NZ9000 strain was grown statically at $30^{\circ} \mathrm{C}$ in M17 broth (BD Difco, Becton, Dickinson and Company, Sparks, MD) supplemented with $0.5 \%$ glucose (GM17). Genetically modified strains of NZ9000 (gmNZ9000) were grown in GM17 with $10 \mu \mathrm{g} / \mathrm{mL}$ chloramphenicol (GM17cm).

\section{Construction of gmNZ9000}

General molecular cloning techniques were performed using previously described methods (Sambrook and Russell, 2001). All restriction enzymes were purchased from Takara Bio (Kusatsu, Shiga, Japan). Primers were synthesized by Eurofins Genomics (Tokyo, Japan) and are listed in Table 1 . Polymerase chain reactions were performed with KOD-Plus-Neo DNA polymerase (Toyobo, Osaka, Japan). After initial denaturation for 5 min at $95^{\circ} \mathrm{C}$, step-down PCR was performed in 3 steps as follows: 5 cycles of $[0.5$-min denaturation at $95^{\circ} \mathrm{C}, 0.5$-min annealing at $65^{\circ} \mathrm{C}$, and 0.5 -min extension at $\left.72^{\circ} \mathrm{C}\right], 10$ cycles of $\left[0.5 \mathrm{~min}\right.$ at $95^{\circ} \mathrm{C}, 0.5 \mathrm{~min}$ at $60^{\circ} \mathrm{C}$, and $0.5 \mathrm{~min}$ at $\left.72^{\circ} \mathrm{C}\right]$, followed by 25 cycles of $[0.5$ min at $95^{\circ} \mathrm{C}, 0.5 \mathrm{~min}$ at $55^{\circ} \mathrm{C}$, and $0.5 \mathrm{~min}$ at $\left.72^{\circ} \mathrm{C}\right]$. A final extension step at $72^{\circ} \mathrm{C}$ for 3 min terminated the program. The strategy for constructing the gene expression vector for the GFP-bLF fusion is shown in Figure 1. Each constructed plasmid was analyzed by DNA sequencing (performed by Eurofins Genomics) and was consistent with the predicted sequence (data not shown).

We attempted to establish a gene expression platform for the GFP fused-target protein. A dehydrofolate reductase (DHFR) coding sequence was amplified from pQE-40 plasmid (Qiagen, Tokyo, Japan) using a primer pair (EcoRI-DHFR F, XhoI-DHFR R). The resulting PCR product was further amplified with a primer pair (XbaI-His-tag-DHFR F, HindIII-FXa-DHFR R) by PCR, and the DNA fragment was subcloned into pTAKN-2 to generate pTAKN-2-HDF. A region of the His-tag-DHFR-Factor Xa recognition site (FXa) on pTAKN-2-HDF was amplified by PCR with a primer pair (NcoI-His-tag F, BamHI-FXa R) and cloned into pNZ8148\#2:CYT to provide pNZ8148\#2:CYT-HDF. The DHFR coding region on pNZ8148\#2:CYT-HDF was removed using restriction enzymes (EcoRI, XhoI) 
Table 1. Primer sequences used in this study

\begin{tabular}{llc}
\hline Primer name & Sequence $\left(5^{\prime}\right.$ to $\left.3^{\prime}\right)$ & $\begin{array}{c}\text { Length } \\
(\mathrm{bp})\end{array}$ \\
\hline BamHI-FXa R & CGGGATCCCCTTCCCTCGATACCAGA & 27 \\
EcoRI-DHFR F & CCGGAATTCATGGTTCGACCATTGAACTC & 29 \\
EcoRI-GFP F & CCGGAATTCGTATCTAAGGGAGAAGAACT & 29 \\
HindIII-FXa-DHFR R & CCCAAGCTTCCTTCCCTCGATACCAGATCCAGAGCCAGATCCCTCGAGTTTCTTCTCGTAGAC & 63 \\
NcoI-His-tag F & CATGCCATGGAAAGAGGATCGCATCACCATC & 31 \\
XbaI-His-tag-DHFR F & GCTCTAGAGGATCGCATCACCATCACCATCACGAATTCATGGTTCGACCATTG \\
XhoI-DHFR R & CCCGCTCGAGTTTCTTCTCGTAGACTTCAAAC & 53 \\
XhoI-GFP R & CCGCTCGAGTTTATATAACTCATCCATGCC & 32 \\
\hline
\end{tabular}

A

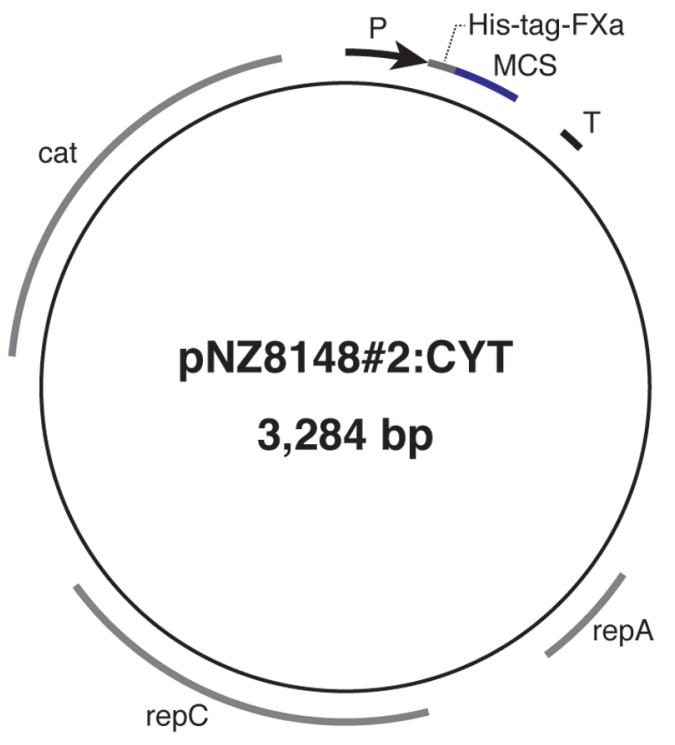

B

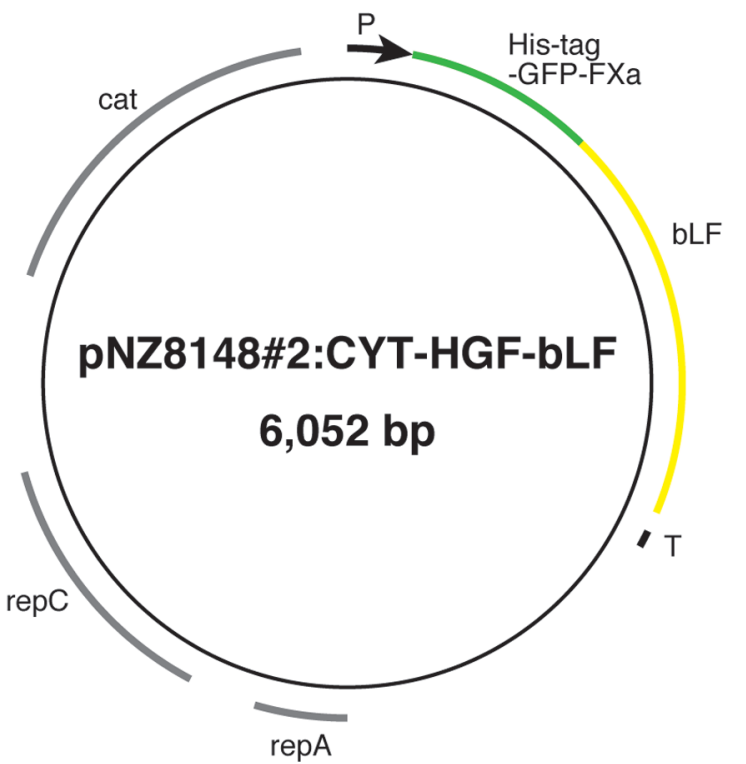

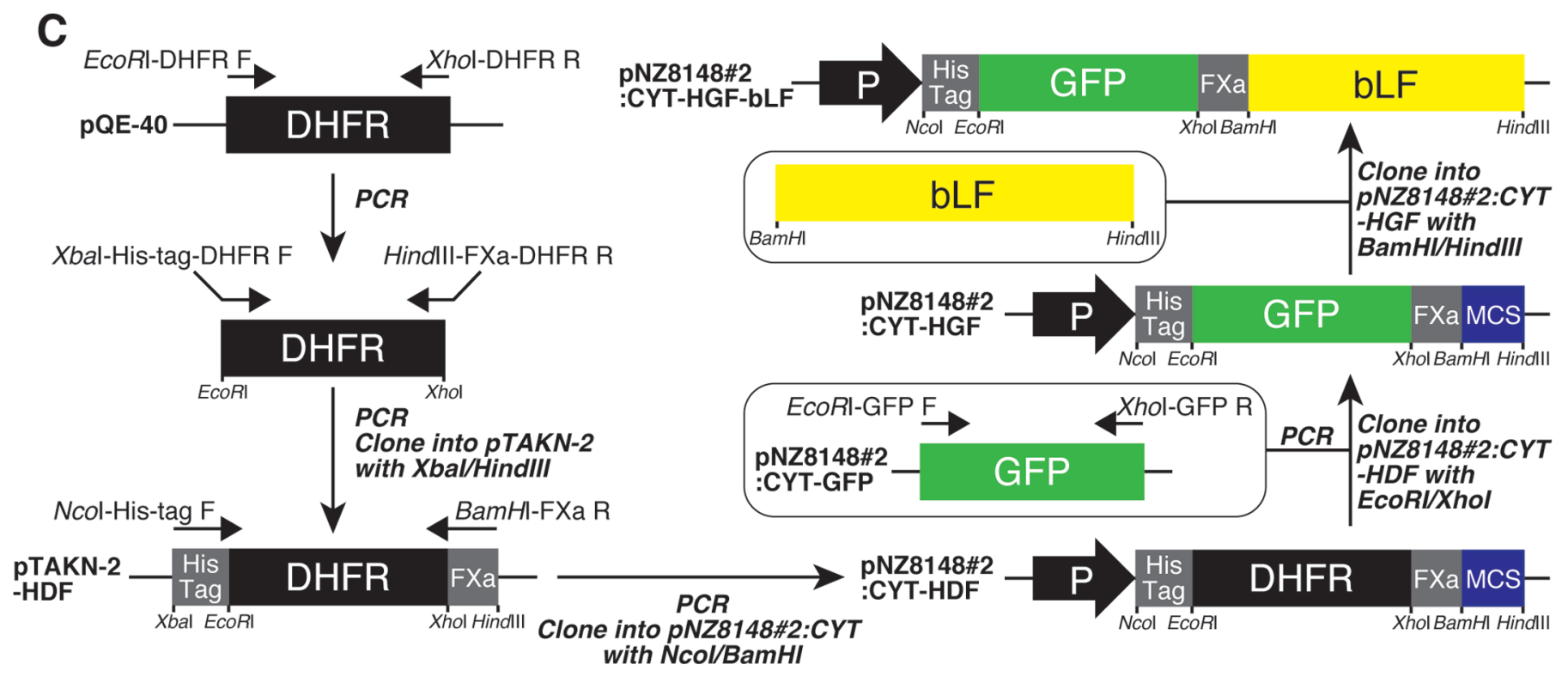

Figure 1. Strategy for the construction of a gene expression vector for a green fluorescent protein-bovine lactoferrin (GFP-bLF) fusion. Based on the lactococcal plasmid pNZ8148\#2:CYT (A), a GFP-bLF gene expression vector (pNZ8148\#2:CYT-HGF-bLF, B) was constructed as described in $(\mathrm{C})$ and in the Materials and Methods section. $\mathrm{P}=$ nisin A promoter; His-tag $=$ hexahistidine tag; FXa = Factor Xa recognition site; $\mathrm{MCS}=$ multiple cloning site; $\mathrm{T}=$ terminator; rep $=$ replication gene; cat $=$ chloramphenicol acetyltransferase gene; DHFR $=$ dehydrofolate reductase. Color version available online. 
to introduce a GFP coding sequence, and was then amplified from pNZ8148\#2:CYT-GFP (Shigemori et al., 2013) with a primer pair (EcoRI-GFP F, XhoI-GFP $\mathrm{R}$ ) by PCR. The resulting plasmid was designated as pNZ8148\#2:CYT-HGF.

A 2,067-bp sequence coding for mature bLF (GenBank accession number: L19981.1, http://www.ncbi. nlm.nih.gov/nuccore/L19981.1) was synthesized and cloned into pTAKN-2 by Eurofins Genomics after codon optimization for Lactococcus lactis ssp. cremoris MG1363. The DNA fragment coding for bLF was excised from the plasmid using restriction enzymes ( $\mathrm{Bam}$ HI, HindIII), and was then introduced into BamHI/ HindIII-digested pNZ8148\#2:CYT-HGF. The resulting plasmid, designated as pNZ8148\#2:CYT-HGF-bLF, was electroporated into NZ9000 using a Gene Pulser Xcell electroporation system (Bio-Rad Laboratories, Hercules, CA) according to the manufacturer's instructions, generating NZ-GFP-bLF.

\section{Induction of Gene Expression}

Gene expression by gmNZ9000 was induced in 2- or $50-\mathrm{mL}$ scale cultures. The experimental procedures were performed according to previously described methods (Shigemori et al., 2013, 2014). Briefly, an overnight culture of gmNZ9000 was inoculated at $1 / 20$ dilution into fresh $\mathrm{GM} 17 \mathrm{~cm}$ broth and incubated at $30^{\circ} \mathrm{C}$ until the culture reached an optical density at $600 \mathrm{~nm}\left(\mathbf{O D}_{600}\right)$ of 0.4. Gene expression was then induced with 1.25 $\mathrm{ng} / \mathrm{mL}$ nisin (MoBiTec) for 0 to $24 \mathrm{~h}$ at $30^{\circ} \mathrm{C}$. Some experiments omitted nisin supplementation. The bacterial cells were then harvested by centrifugation $(8,000$ $\left.\times g, 4^{\circ} \mathrm{C}, 5 \mathrm{~min}\right)$ and washed with ice-cold Tris-buffered saline (TBS: $50 \mathrm{~m} M$ Tris-HCl, $140 \mathrm{~m} M$ sodium chloride, $\mathrm{pH}$ 8.0) or PBS (137 $\mathrm{m} M$ sodium chloride, $2.7 \mathrm{mM}$ potassium chloride, $10 \mathrm{~m} M$ disodium hydrogen phosphate, $1.76 \mathrm{mM}$ potassium dihydrogen phosphate, $\mathrm{pH}$ 7.4). The TBS-washed cells were used for fluorescence analyses and Western blotting. The PBS-washed pellet was resuspended in PBS and stored at $-20^{\circ} \mathrm{C}$ until used to stimulate RAW 264.7 cells.

\section{Confocal Laser Scanning Microscopy}

A freshly prepared TBS-washed bacterial pellet was suspended in $400 \mu \mathrm{L}$ of TBS; then, $10 \mu \mathrm{L}$ was placed on a microscope slide and observed with a confocal laser scanning microscope (FluoView FV1000, Olympus Corp., Tokyo, Japan).

\section{Preparation of Cellular Extracts}

The bacterial cellular pellet from a 2 -mL culture was suspended in $400 \mu \mathrm{L}$ of TBS containing protease inhibi- tor cocktail (PIC, Roche Diagnostics, Indianapolis, IN; TBS-PIC). Bacterial cells were then disrupted using a bead beater (Beads Crasher $\mu \mathrm{T}-12$, Taitec, Saitama, Japan) with glass beads (0.2-mm diameter; AS ONE, Osaka, Japan). The soluble fraction was collected by centrifugation at $20,400 \times g$ at $4^{\circ} \mathrm{C}$ for $15 \mathrm{~min}$. Protein concentrations of the cellular extracts were measured with a bicinchoninic acid (BCA) Protein Assay Kit (Thermo Fisher Scientific, Rockford, IL). Samples were stored at $-80^{\circ} \mathrm{C}$.

\section{Western Blotting}

Cellular extracts were boiled in an equal volume of $2 \times$ sample buffer for $5 \mathrm{~min}$. A 2.5- $\mu \mathrm{g}$ aliquot was resolved by SDS-polyacrylamide gel $(7.5 \% \mathrm{vol} / \mathrm{vol}$ polyacrylamide) electrophoresis, and then the separated proteins were transferred from the gel onto a polyvinylidene difluoride membrane (GE Healthcare, Amersham, UK). Western blotting was performed with mouse anti-Histag antibody (Ab) $(1 / 1,000,652501$; BioLegend, San Diego, CA), mouse anti-GFP Ab (1/1,000, 668205; BioLegend), or goat anti-bLF Ab (1/1,000, A10-126A; Bethyl Laboratories, Montgomery, TX), followed by incubation with horseradish peroxidase (HRP)-conjugated goat anti-mouse IgG Ab (1/5,000, A4416; SigmaAldrich, St. Louis, MO) or HRP-conjugated donkey anti-goat IgG Ab $(1 / 5,000$, HAF109; R\&D Systems, Minneapolis, MN). The resulting blots were treated with ECL Prime Western Blotting Detection Reagent (GE Healthcare) and signals were detected using a lumino image analyzer (ImageQuant LAS 4000 mini, GE Healthcare).

\section{Fluorometric Analysis of Cellular Extracts}

The fluorescence intensity of a $100-\mu \mathrm{L}$ aliquot of cellular extract diluted to $200 \mu \mathrm{g}$ of protein $/ \mathrm{mL}$ with TBS-PIC was analyzed using a Fluoroskan Ascent FL Microplate Fluorometer (Thermo Fisher Scientific) by excitation at $485 \mathrm{~nm}$ and emission at $538 \mathrm{~nm}$.

\section{ELISA for Quantification of bLF}

The bLF concentrations of cellular extracts were quantified using a modification of a standard ELISA technique (Voller et al., 1978). Briefly, flat-bottomed, 96-well immune plates (Nunc MaxiSorp, Nunc, Roskilde, Denmark) were coated by overnight incubation at $4^{\circ} \mathrm{C}$ with TBS as a blank, various concentrations (80 to $5,000 \mathrm{pg} / \mathrm{mL}$ ) of bLF (Wako Pure Chemical Industries Ltd., Osaka, Japan) as a standard protein, or the cellular extracts $(200 \mu \mathrm{g}$ of protein $/ \mathrm{mL})$ prepared from 5 independent experiments. The plates were washed with TBS containing $0.05 \%$ Tween-20 (TBS-T) and 
then saturated with TBS-T by incubation for $30 \mathrm{~min}$ at room temperature (RT). The plate-immobilized compounds were reacted with goat anti-bLF Ab (1/1,000, A10-126A; Bethyl Laboratories) for $1 \mathrm{~h}$ at RT. After washing the plates with TBS-T, the resulting complexes were further reacted with HRP-conjugated donkey anti-goat IgG Ab (1/5,000, HAF109; R\&D Systems) for $1 \mathrm{~h}$ at RT, and then visualized by a HRP-substrate (3,3',5,5'-tetramethylbenzidine; Nacalai Tesque, Kyoto, Japan) reaction for $15 \mathrm{~min}$ at $\mathrm{RT}$. The reaction was stopped with $2 N$ sulfuric acid and absorbance was measured at $450 \mathrm{~nm}$. In all experiments, we confirmed good correlation between bLF concentration and absorbance at $450 \mathrm{~nm}\left(\mathrm{R}^{2}>0.95\right)$.

\section{Culture Conditions for RAW 264.7 Cells}

The mouse macrophage cell line RAW 264.7 was purchased from the American Type Culture Collection (Manassas, VA) and maintained in Dulbecco's modified Eagle's medium (DMEM; Gibco, Thermo Fisher Scientific) containing 10\% fetal bovine serum (HyClone, GE Healthcare), $100 \mathrm{U} / \mathrm{mL}$ penicillin, and $100 \mu \mathrm{g} /$ $\mathrm{mL}$ streptomycin (Nacalai Tesque) (complete DMEM) at $37^{\circ} \mathrm{C}$ in a humidified incubator supplied with $5 \%$ carbon dioxide. The RAW 264.7 cells were subcultured every 2 or $3 \mathrm{~d}$.

\section{Real-Time Quantitative PCR}

The RAW 264.7 cells were seeded into a 24-well cell culture plate at a concentration of $2 \times 10^{5}$ cells $/ \mathrm{mL}$ (total $1 \mathrm{~mL} /$ well). After 24-h culture, the culture supernatant was replaced with $1 \mathrm{~mL}$ of fresh complete DMEM containing gmLAB cells $\left(4 \times 10^{7} \mathrm{cfu}\right.$, mouse cell:bacteria $=1: 200$ ) and the culture was incubated for another $24 \mathrm{~h}$. Cells were then stimulated apically with LPS from E. coli O111:B4 (InvivoGen, San Diego, CA) at a final concentration of $100 \mathrm{ng} / \mathrm{mL}$ for $3 \mathrm{~h}$. Total RNA was isolated from the cells using a commercial RNA isolation kit (NucleoSpin RNA, Macherey-Nagel, Düren, Germany) according to the manufacturer's instructions. The purified total RNA was reverse-transcribed using PrimeScript RT Master Mix (Takara Bio) according to the manufacturer's instructions. Real-time quantitative PCR analysis was performed with a 10-fold diluted solution of the cDNA reaction mixture, SYBR Premix Ex Taq (Takara Bio), and mouse $\beta$-actin- or cytokine-specific primers (Takara Bio) as described previously (Ito et al., 2012).

\section{Cytokine ELISA}

For ELISA, RAW 264.7 cells $\left(2 \times 10^{5}\right)$ were grown in $2 \mathrm{~mL}$ of complete DMEM in a 6 -well cell culture plate for $24 \mathrm{~h}$. The culture medium was exchanged with $2 \mathrm{~mL}$ of fresh complete DMEM containing gmLAB cells $(4 \times$ $10^{7} \mathrm{cfu}$, mouse cell:bacteria $\left.=1: 200\right)$. The culture was incubated for $24 \mathrm{~h}$, and then $100 \mathrm{ng}$ of LPS from E. coli O111:B4 (InvivoGen) was added apically. After incubation for $24 \mathrm{~h}$, the culture supernatant was recovered and stored at $-80^{\circ} \mathrm{C}$. Plate-adhered cells were washed with PBS and lysed with CelLytic M (Sigma-Aldrich) supplemented with PIC (Sigma-Aldrich) as described previously (Wang et al., 2015). The concentrations of IL- $1 \alpha$ in the cellular lysates, and of IL- 6 and tumor necrosis factor (TNF)- $\alpha$ in the culture supernatants, were measured using ELISA kits (IL-1 $\alpha$ : BioLegend; IL-6: R\&D Systems; TNF- $\alpha$ : eBioscience, San Diego, CA) according to the manufacturers' instructions.

\section{Statistical Analysis}

Statistical analyses were performed using a statistical software package (GraphPad Prism7, GraphPad Software Inc., La Jolla, CA). One-way ANOVA with the post hoc Tukey-Kramer test was used to determine the significance of differences.

\section{RESULTS}

\section{Nisin-Induced Production of a Fusion Protein of GFP and bLF in NZ-GFP-bLF}

Cellular extracts from the nisin-induced and noninduced gmNZ9000 strains (i.e., NZ-VC and NZ-GFPbLF) were subjected to Western blotting. Western blotting using antibodies against His-tag (Figure 2A), bLF (Figure 2B), or GFP (Figure 2C) showed a band corresponding to a fusion of GFP and bLF (rGFP-bLF) only in cellular extracts of nisin-induced NZ-GFP-bLF. No common bands were observed in cellular extracts from nisin-induced NZ-VC (vector control) and noninduced gmNZ9000 strains (Figure 2).

\section{Functional Analysis of GFP in the rGFP-bLF Fusion Protein}

To verify the functionality of GFP in the fusion protein produced by NZ-GFP-bLF, GFP fluorescence in nisin-induced gmNZ9000 strain was analyzed by 2 methods: confocal laser scanning microscopy (Figure $3 \mathrm{~A}$ to F) and fluorometric analysis (Figure 3G). Green fluorescent protein fluorescence was observed in nisininduced NZ-GFP-bLF but not in nisin-induced NZ-VC cells (Figure $3 \mathrm{~A}$ to $\mathrm{F}$ ). The fluorescence intensity of GFP in cellular extracts of nisin-induced NZ-GFP-bLF was markedly higher than that of nisin-induced NZ-VC. 
A

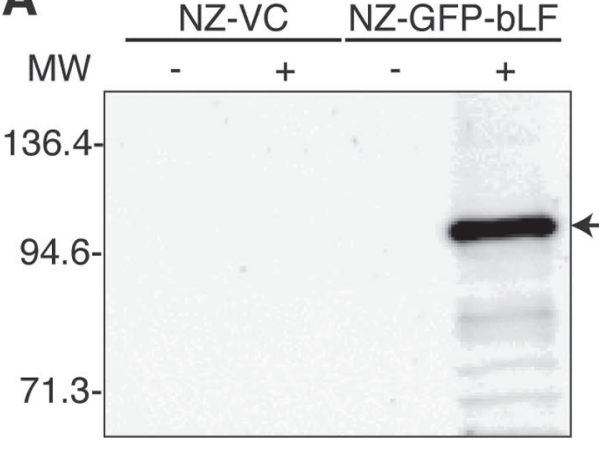

B

$\mathrm{MW} \frac{\mathrm{NZ}-\mathrm{VC}}{-}+\frac{\mathrm{NZ}-\mathrm{GFP}-\mathrm{bLF}}{-}$

C

$\mathrm{MW} \frac{\mathrm{NZ}-\mathrm{VC}}{-\mathrm{N}} \frac{\mathrm{NZ}-\mathrm{GFP}-\mathrm{bLF}}{-+}$

136.4

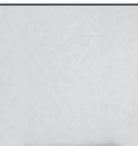

136.4
-94.6
$71.3-$

$+94.6-$
$71.3-$

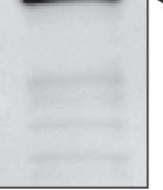

71.3-

Figure 2. Detection of recombinant green fluorescent protein-bovine lactoferrin (rGFP-bLF) fusion. The NZ-VC (vector control) and NZGFP-bLF gmNZ9000 strains were cultured with $(+)$ or without $(-)$ nisin for $3 \mathrm{~h}$. Cellular extracts from the respective cultures were analyzed by Western blotting using specific antibodies against hexahistidine tag (A), bLF (B), or GFP (C). Arrows indicate rGFP-bLF (107.1 kDa). MW $=$ molecular weight.

\section{Characterization of Growth and rGFP-bLF Production with Time by Nisin-Induced NZ-GFP-bLF}

After supplementation of the culture medium with nisin $\left(0 \mathrm{~h}: \mathrm{OD}_{600}=0.4\right.$, early stage exponential phase), the $\mathrm{OD}_{600}$ of the NZ-VC culture increased dramatically in a time-dependent manner until $3 \mathrm{~h}$, and then remained essentially constant (Figure 4). In contrast, the $\mathrm{OD}_{600}$ of the NZ-GFP-bLF culture increased significantly more slowly (Figure 4 ). To verify the change in the production level of rGFP-bLF in NZ-GFP-bLF cells with time, the fluorescence intensities of GFP in cellular extracts of nisin-induced gmNZ9000 were measured at various time points. The relative fluorescence units (RFU) of cellular extracts of NZ-GFP-bLF peaked 2 to $3 \mathrm{~h}$ after nisin induction, then decreased gradually until $24 \mathrm{~h}$ after induction (Figure 4). A similar transition of rGFP-bLF production was observed by Western blotting (data not shown). The levels of rGFPbLF in cellular extracts prepared from nisin-induced (3 h) NZ-GFP-bLF and NZ-VC were analyzed by ELISA with anti-bLF Ab and were $1,544.8 \pm 82.9 \mathrm{pg} / \mathrm{mg}$ of protein and below the detection limit of the ELISA, respectively.
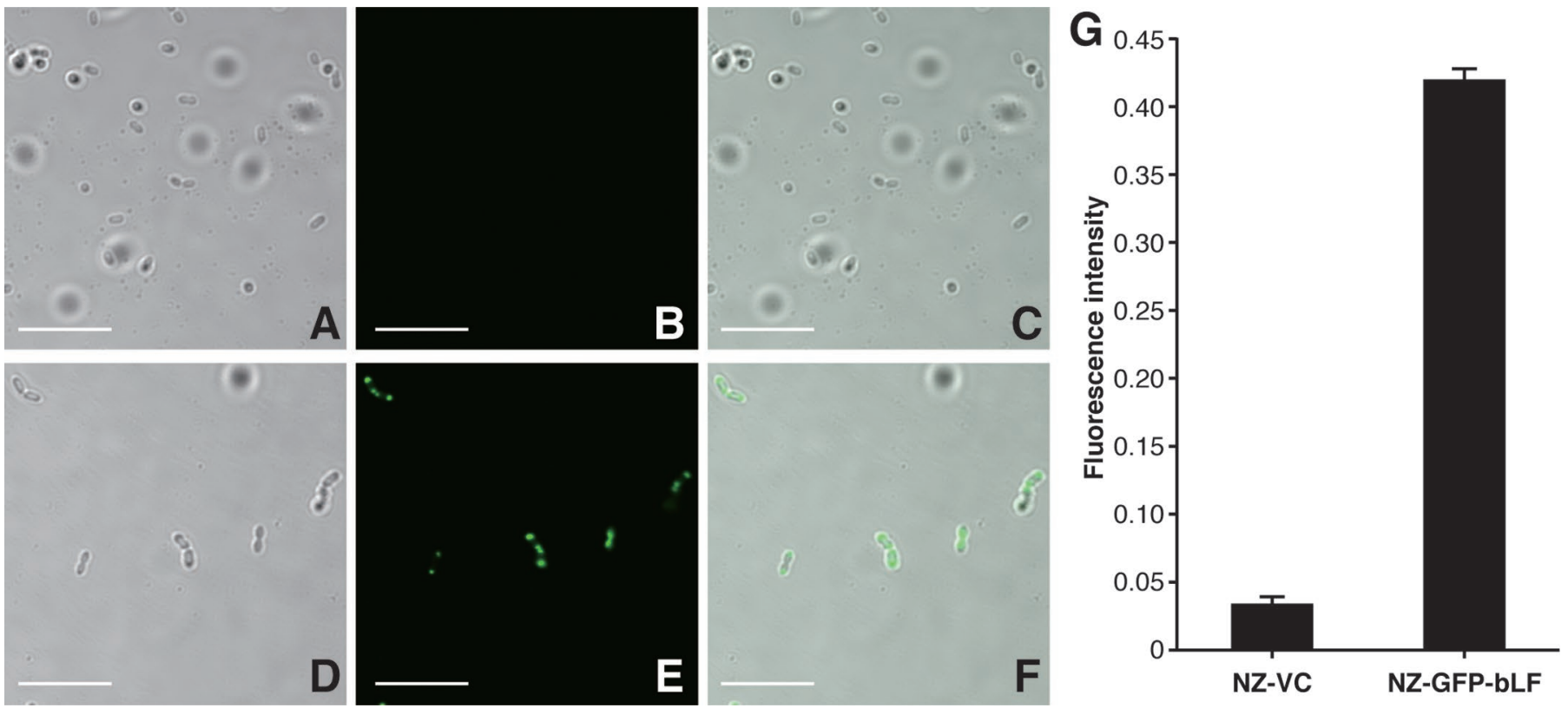

Figure 3. Fluorescence analyses of genetically modified NZ9000 strains. The NZ-VC (vector control; A to C) and NZ-GFP-bLF (green fluorescent protein-bovine lactoferrin fusion; D and E) gmNZ9000 strains were cultured with nisin for $3 \mathrm{~h}$. The resulting cells were observed using a confocal laser scanning microscope under visible $(\mathrm{A}, \mathrm{D})$ and fluorescent light $(\mathrm{B}, \mathrm{E})$, and then merged images $(\mathrm{C}, \mathrm{F})$ were generated. Bar $=$ $10 \mu \mathrm{m}$. Cellular extracts from nisin-induced genetically modified NZ9000 were analyzed using a fluorescence microplate reader $(\mathrm{G})$. Data are expressed as mean $+\mathrm{SD}(\mathrm{n}=3)$. 


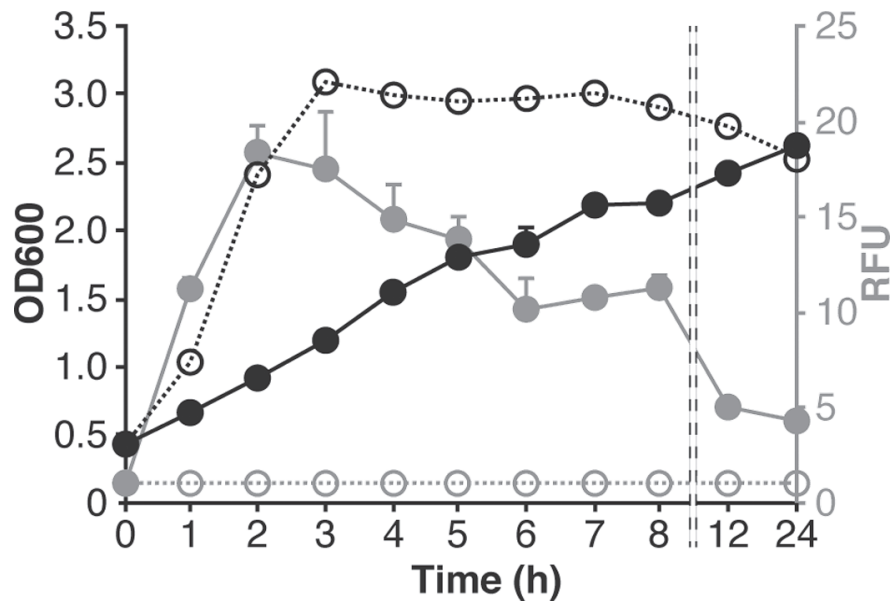

Figure 4. Changes in the optical density and green fluorescent protein (GFP) fluorescence of NZ-VC (vector control) and NZ-GFP-bLF (green fluorescent protein-bovine lactoferrin fusion) genetically modified NZ9000 strain cultures with time. The NZ-VC (dashed lines) and NZ-GFP-bLF (solid lines) strains were cultured with nisin for 0 to 24 $\mathrm{h}$. The optical density at $600 \mathrm{~nm}\left(\mathrm{OD}_{600}\right)$ of the cultures (black lines) was measured at the indicated time points. The fluorescence intensities of the cellular extracts were measured at the indicated time points, and the relative fluorescence units (RFU, gray lines) were calculated as relative values compared with NZ-VC. Data are expressed as mean $+\mathrm{SD}(\mathrm{n}=2)$.

\section{Preventive Effects of Nisin-Induced NZ-GFP-bLF on Proinflammatory Cytokine Expression in LPS-Stimulated RAW 264.7 Cells}

The anti-inflammatory property of NZ-GFP-bLF in vitro was investigated by examining the mRNA expres- sion (Figure 5) and protein production (Figure 6) levels of typical proinflammatory cytokines (IL-1 $\alpha$, IL-6, and TNF- $\alpha$ ) induced by LPS in RAW 264.7 cells prestimulated with nisin-induced NZ-VC or NZ-GFP-bLF for $24 \mathrm{~h}$. The mRNA expression of the 3 cytokines was strongly induced in the NZ-VC-conditioned cells after a 3-h stimulation with LPS (Figure 5), whereas expression was significantly suppressed in the NZ-GFP-bLFconditioned cells $(P<0.01)$. Consistent with mRNA expression, robust production of IL-1 $\alpha$, IL-6, and TNF- $\alpha$ was observed in the NZ-VC-conditioned cells after a 24-h stimulation with LPS and was significantly reduced in the NZ-GFP-bLF-conditioned cells $(P<$ 0.01, Figure 6).

\section{DISCUSSION}

Previously, we developed an expression system for a fusion protein comprising GFP and a food allergen $\left(\alpha_{\mathrm{S}_{1}}\right.$-casein or Fagag1) in NZ9000 (Shigemori et al., 2012, 2013). We demonstrated that the fusion protein exhibited characteristics of both the fluorescent protein and the allergen, suggesting the validity of this system for overexpression of bioactive proteins and easy tracking of recombinant proteins in vitro and in vivo. In the present study, we adopted the GFP fusion system with slight modifications to construct bLF-expressing $L$. lactis. First, we modified the gene expression vector to insert a cleavable linker, FXa, between the GFP gene and a cloning site of the target gene to facilitate purification of the target protein from the fusion. Next, we cloned
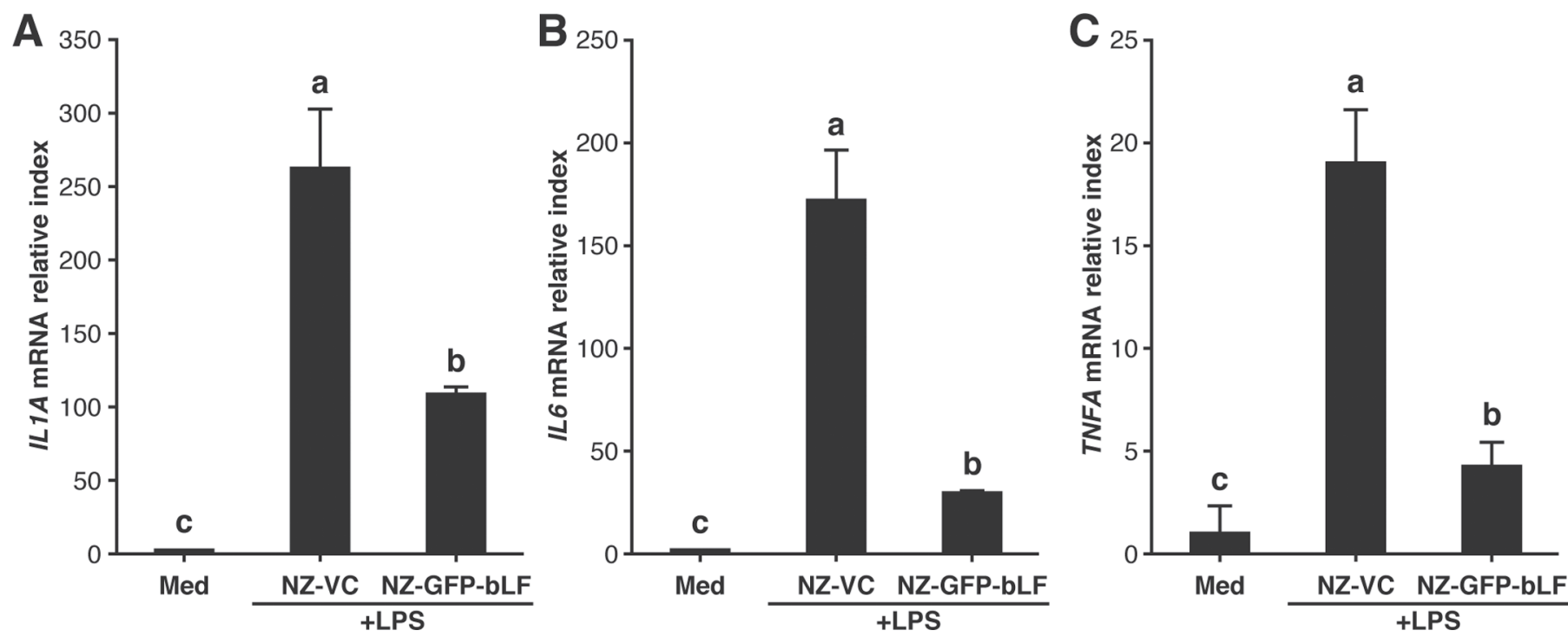

Figure 5. The effect of nisin-induced genetically modified NZ9000 strain (NZ-GFP-bLF) on LPS-induced cytokine mRNA expression in RAW 264.7 cells. The RAW 264.7 cells were pretreated with or without nisin-induced NZ-VC (vector control) or NZ-GFP-bLF (green fluorescent protein-bovine lactoferrin fusion) for $24 \mathrm{~h}$; then, the cells were apically stimulated with or without LPS for $3 \mathrm{~h}$. Interleukin-1 $\alpha$, IL-6, and tumor necrosis factor (TNF)- $\alpha$ mRNA expression was measured by real-time quantitative PCR. Data are expressed as mean $+\mathrm{SD}(\mathrm{n}=3)$. Bars with different letters $(\mathrm{a}, \mathrm{b}, \mathrm{c})$ were significantly different $(P<0.01)$. Med $=$ medium control. 

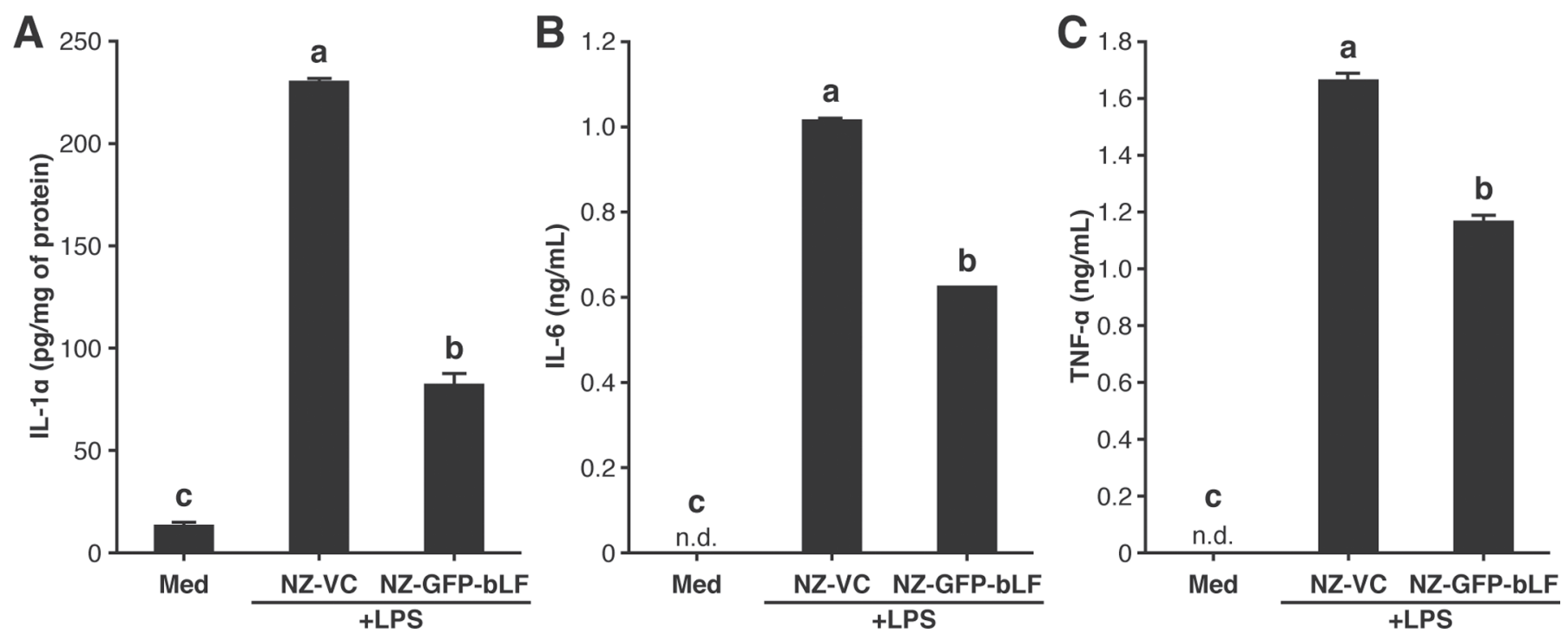

Figure 6. The effect of genetically modified NZ9000 strain NZ-GFP-bLF on LPS-induced cytokine production in RAW 264.7 cells. The RAW 264.7 cells were pretreated with or without nisin-induced NZ-VC (vector control) or NZ-GFP-bLF (green fluorescent protein-bovine lactoferrin fusion) for $24 \mathrm{~h}$; then, the cells were apically stimulated with or without LPS for $24 \mathrm{~h}$. Interleukin-1 $\alpha$ production in the cells and IL-6 and tumor necrosis factor (TNF)- $\alpha$ secretion into the culture medium were measured by ELISA. Data are expressed as mean + SD $(n=3)$. Bars with different letters $(\mathrm{a}, \mathrm{b}, \mathrm{c})$ were significantly different $(P<0.01)$. Med $=$ medium control; n.d. = nondetectable.

a codon-optimized bLF-coding sequence downstream of FXa on pNZ8148\#2:CYT-HGF to generate an expression vector for the GFP-bLF fusion. Finally, we constructed a gmNZ9000 strain (NZ-GFP-bLF) harboring the GFP-bLF expression vector pNZ8148\#2:CYTHGF-bLF. Western blotting showed that NZ-GFP-bLF produced a recombinant protein with reactivity to antiHis tag, anti-GFP, and anti-bLF antibodies, and a molecular mass corresponding to rGFP-bLF. This protein was expressed following nisin stimulation. Additionally, we demonstrated by 2 fluorescence assays that rGFP$\mathrm{bLF}$ is a fluorescent protein.

We monitored time-dependent changes in the production level of rGFP-bLF based on GFP RFU. Consistent with our previous findings (Shigemori et al., 2012, 2013), rGFP-bLF production peaked 2 to $3 \mathrm{~h}$ after nisin supplementation of the culture medium, and then gradually decreased after $3 \mathrm{~h}$. The reduction in RFU may be associated with degradation of rGFP-bLF by endogenous proteases in L. lactis (Le Loir et al., 2005; Morello et al., 2008). Interestingly, the maximum value of RFU obtained from NZ-GFP-bLF (about 18) was approximately 6 -fold higher (RFU against NZ-VC $=\sim 3)$ than that observed previously in GFP-allergen fusion-producing gmNZ9000 strains (Shigemori et al., 2012, 2013). Although a simple comparison is impossible because the GFP-fused proteins have different structures and properties, insertion of the FXa linker may increase the quality or quantity of the recombinant protein. The growth of nisin-induced NZ-GFP-
bLF was significantly slower than that of nisin-induced NZ-VC and the previously established gmNZ9000 strains producing GFP-fused $\alpha_{\mathrm{S} 1}$-casein (Shigemori et al., 2012), GFP-fused Fagag1 (Shigemori et al., 2013), or antibody fragment against mouse IL-6 (Shigemori et al., 2017). This result suggests that the bLF moiety in rGFP-bLF is bioactive, given that L. lactis is susceptible to the antimicrobial activity of bLF (Andrés and Fierro, 2010).

Sepsis is the life-threatening dysfunction of an organ caused by a dysregulated host response to infection (Singer et al., 2016). Lipopolysaccharide is a main pathogenic factor in sepsis caused by gram-negative bacterial infection and induces excessive immune responses such as "cytokine storm" through toll-like receptor 4 (TLR4) on the host cells, including macrophages, toward inflammation and injury (Park and Lee, 2013). Lactoferrin reduces LPS-induced immune activation in vitro and in an animal model through its ability to inhibit the interaction of LPS with TLR4, and directly modulates cytokine production in monocytic cells (Latorre et al., 2010). Therefore, we investigated the effects of NZ-GFP-bLF on the mRNA expression and protein production of proinflammatory cytokines (IL$1 \alpha$, IL-6, and TNF- $\alpha$ ) in LPS-stimulated RAW 264.7 macrophages. The results of these assays showed that NZ-GFP-bLF exerted a significant preventive effect on cytokine expression and production in LPS-stimulated RAW 264.7 cells compared with NZ-VC $(P<0.01)$. These results clearly demonstrate that the recombinant 
protein produced by NZ-GFP-bLF has bioactivity for LPS-induced immune activation.

In conclusion, we constructed a new gmNZ9000 strain (NZ-GFP-bLF) that produces an rGFP-bLF fusion protein. We showed, using functional studies, that rGFP-bLF has the desired bioactivities (i.e., fluorescence and antibacterial/anti-inflammation properties). Furthermore, we clearly demonstrated the beneficial effect of NZ-GFP-bLF for preventing LPS-induced cytokine expression and production in RAW 264.7 macrophages. These results suggest that NZ-GFP-bLF is attractive as a preventive agent for gram-negative sepsis and an efficient cell factory for bLF production. Further studies are needed to demonstrate the preventive effect and mechanism(s) of NZ-GFP-bLF on LPS-induced insults in animal models. The GFP fluorescence of the fusion protein will be helpful in those studies.

\section{ACKNOWLEDGMENTS}

The authors thank the Faculty of Agriculture, Shinshu University (Kamiina, Nagano, Japan) for funding this study. The authors declare that they have no conflict of interest.

\section{REFERENCES}

Andrés, M. T., and J. F. Fierro. 2010. Antimicrobial mechanism of action of transferrins: Selective inhibition of $\mathrm{H}^{+}$-ATPase. Antimicrob. Agents Chemother. 54:4335-4342.

Cano-Garrido, O., J. Seras-Franzoso, and E. Garcia-Fruitos. 2015. Lactic acid bacteria: Reviewing the potential of a promising delivery live vector for biomedical purposes. Microb. Cell Fact. 14:137.

Farnaud, S., and R. W. Evans. 2003. Lactoferrin-a multifunctional protein with antimicrobial properties. Mol. Immunol. 40:395-405.

Ito, Y., S. Shigemori, T. Sato, T. Shimazu, K. Hatano, H. Otani, H. Kitazawa, and T. Shimosato. 2012. Class I/II hybrid inhibitory oligodeoxynucleotide exerts Th1 and Th2 double immunosuppression. FEBS Open Bio 3:41-45.

Kruzel, M. L., Y. Harari, C. Y. Chen, and G. A. Castro. 2000. Lactoferrin protects gut mucosal integrity during endotoxemia induced by lipopolysaccharide in mice. Inflammation 24:33-44.

Latorre, D., P. Puddu, P. Valenti, and S. Gessani. 2010. Reciprocal interactions between lactoferrin and bacterial endotoxins and their role in the regulation of the immune response. Toxins (Basel) 2:54-68.

Le Loir, Y., V. Azevedo, S. C. Oliveira, D. A. Freitas, A. Miyoshi, L. G. Bermudez-Humaran, S. Nouaille, L. A. Ribeiro, S. Leclercq, J. E. Gabriel, V. D. Guimaraes, M. N. Oliveira, C. Charlier, M. Gautier, and P. Langella. 2005. Protein secretion in Lactococcus lactis: An efficient way to increase the overall heterologous protein production. Microb. Cell Fact. 4:2.

Lee, W. J., J. L. Farmer, M. Hilty, and Y. B. Kim. 1998. The protective effects of lactoferrin feeding against endotoxin lethal shock in germfree piglets. Infect. Immun. 66:1421-1426.

Manzoni, P., M. Rinaldi, S. Cattani, L. Pugni, M. G. Romeo, H. Messner, I. Stolfi, L. Decembrino, N. Laforgia, F. Vagnarelli, L. Memo, L. Bordignon, O. S. Saia, M. Maule, E. Gallo, M. Mostert, C. Magnani, M. Quercia, L. Bollani, R. Pedicino, L. Renzullo, P. Betta, F. Mosca, F. Ferrari, R. Magaldi, M. Stronati, and D. Farina. 2009. Bovine lactoferrin supplementation for prevention of late-onset sepsis in very low-birth-weight neonates: A randomized trial. JAMA 302:1421-1428.
Masson, P. L., and J. F. Heremans. 1971. Lactoferrin in milk from different species. Comp. Biochem. Physiol. B 39:119-129.

Morello, E., L. G. Bermudez-Humaran, D. Llull, V. Sole, N. Miraglio, P. Langella, and I. Poquet. 2008. Lactococcus lactis, an efficient cell factory for recombinant protein production and secretion. J. Mol. Microbiol. Biotechnol. 14:48-58.

Park, B. S., and J. O. Lee. 2013. Recognition of lipopolysaccharide pattern by TLR4 complexes. Exp. Mol. Med. 45:e66.

Sambrook, J., and D. Russell. 2001. Molecular Cloning: A Laboratory Manual. 3rd ed. Cold Spring Harbor Laboratory, Cold Spring Harbor, NY

Sherman, M. P., S. H. Bennett, F. F. Hwang, and C. Yu. 2004. Neonatal small bowel epithelia: enhancing anti-bacterial defense with lactoferrin and Lactobacillus GG. Biometals 17:285-289.

Shigemori, S., M. Ihara, T. Sato, Y. Yamamoto, S. Nigar, T. Ogita, and T. Shimosato. 2017. Secretion of an immunoreactive singlechain variable fragment antibody against mouse interleukin 6 by Lactococcus lactis. Appl. Microbiol. Biotechnol. 101:341-349.

Shigemori, S., K. Oshiro, P. Wang, Y. Yamamoto, Y. Wang, T. Sato, Y. Uyeno, and T. Shimosato. 2014. Generation of dipeptidyl peptidase-IV-inhibiting peptides from $\beta$-lactoglobulin secreted by Lactococcus lactis. BioMed Res. Int. 2014:393598.

Shigemori, S., and T. Shimosato. 2017. Applications of genetically modified immunobiotics with high immunoregulatory capacity for treatment of inflammatory bowel diseases. Front. Immunol. 8:22.

Shigemori, S., T. Watanabe, K. Kudoh, M. Ihara, S. Nigar, Y. Yamamoto, Y. Suda, T. Sato, H. Kitazawa, and T. Shimosato. 2015. Oral delivery of Lactococcus lactis that secretes bioactive heme oxygenase-1 alleviates development of acute colitis in mice. Microb. Cell Fact. 14:189.

Shigemori, S., S. Yonekura, T. Sato, M. Nakanishi, H. Otani, and T. Shimosato. 2012. Expression of a biologically active GFP$\alpha_{\mathrm{S1} 1}$-casein fusion protein in Lactococcus lactis. Curr. Microbiol. 64:569-575.

Shigemori, S., S. Yonekura, T. Sato, H. Otani, and T. Shimosato. 2013. Expression of the immunoreactive buckwheat major allergenic storage protein in Lactococcus lactis. Appl. Microbiol. Biotechnol. 97:3603-3611.

Singer, M., C. S. Deutschman, C. W. Seymour, M. Shankar-Hari, D Annane, M. Bauer, R. Bellomo, G. R. Bernard, J. D. Chiche, C. M. Coopersmith, R. S. Hotchkiss, M. M. Levy, J. C. Marshall, G. S. Martin, S. M. Opal, G. D. Rubenfeld, T. van der Poll, J. L. Vincent, and D. C. Angus. 2016. The Third International Consensus Definitions for Sepsis and Septic Shock (Sepsis-3). JAMA 315:801-810.

Siqueiros-Cendón, T., S. Arevalo-Gallegos, B. F. Iglesias-Figueroa, I. A. Garcia-Montoya, J. Salazar-Martinez, and Q. Rascon-Cruz. 2014. Immunomodulatory effects of lactoferrin. Acta Pharmacol. Sin. 35:557-566.

Talukder, M. J., and E. Harada. 2007. Bovine lactoferrin protects lipopolysaccharide-induced diarrhea modulating nitric oxide and prostaglandin E2 in mice. Can. J. Physiol. Pharmacol. 85:200-208.

Tsuji, S., Y. Hirata, F. Mukai, and S. Ohtagaki. 1990. Comparison of lactoferrin content in colostrum between different cattle breeds. J. Dairy Sci. 73:125-128.

Voller, A., A. Bartlett, and D. E. Bidwell. 1978. Enzyme immunoassays with special reference to ELISA techniques. J. Clin. Pathol. 31:507-520.

Wang, Y., Y. Yamamoto, S. Shigemori, T. Watanabe, K. Oshiro, X. Wang, P. Wang, T. Sato, S. Yonekura, S. Tanaka, H. Kitazawa, and T. Shimosato. 2015. Inhibitory/suppressive oligodeoxynucleotide nanocapsules as simple oral delivery devices for preventing atopic dermatitis in mice. Mol. Ther. 23:297-309.

Wells, J. M., and A. Mercenier. 2008. Mucosal delivery of therapeutic and prophylactic molecules using lactic acid bacteria. Nat. Rev. Microbiol. 6:349-362.

Zagulski, T., P. Lipinski, A. Zagulska, S. Broniek, and Z. Jarzabek. 1989. Lactoferrin can protect mice against a lethal dose of Escherichia coli in experimental infection in vivo. Br. J. Exp. Pathol. 70:697-704. 